\title{
EDITORIAL
}

\section{Oncolytic viruses as platform for multimodal cancer therapeutics: a promising land}

Cancer Gene Therapy (2014) 21, 261-263; doi:10.1038/cgt.2014.31

Oncolytic virus (OV) therapy with T-VEC for patients with advanced melanoma in a successful phase III clinical trial has showcased the potential of this novel modality of cancer treatment. OVs kill cancer cells and associated stromal cells via multimodal mechanisms, killing directly by infection and oncolysis, indirectly by vasculature-targeting, bystander effect and elicited antitumor immunity. For most OVs, the antitumor immunity elicited by the OV contributes to, or even plays essential roles in the overall efficacy of the oncolytic virotherapy (OVT). ${ }^{1-3}$

We and others believe that OVT will be much more efficacious when combined with other modalities of cancer treatments (Figure 1). ${ }^{4}$ In this Editorial, we would like to expand and update this idea of multimodal therapy using OVs as a fruitful platform. Generally speaking, multimodal therapy is more effective than single agent for cancer treatment. In 1998, Chiocca and colleagues 5 pioneered such an approach by showing that prodrug-enzyme therapy can be integrated into OVT. They had designed a herpes simplex viruses type 1 (HSV-1) with an inactivated gene for viral ribonucleotide reductase (ICP6) to target tumor cells with upregulated mammalian ribonucleotide reductase $(m R R)$, an enzyme whose expression is regulated by the p16/pRB tumor suppressor pathway. A recombinant HSV-1 was generated with ICP6 gene-deletion and expressing rat CYP2B1 transgene, whose product can activate the prodrugs cyclophosphamide and ifosfamide. The recombinant HSV replicated selectively in mRRexpressing cancer cells. Addition of cyclophosphamide potentiated oncolytic effects against cultured tumor cells and subcutaneous tumor xenografts in athymic mice. ${ }^{5}$ This and many other studies later on have demonstrated the potentials of such combinatorial strategies in both preclinical studies and clinical trials.

\section{COMBINATION WITH CHEMOTHERAPY OR RADIATION THERAPY}

We and many others have explored combining OVs with chemotherapy. The first approved OV for clinical use in the world is to be used in conjunction with chemotherapy (Fluorouracil and cisplatin) for head and neck cancer. Two recent studies represent the latest developments in the field. OVs such as HSV can mediate DNA damage responses, and the combination with a chemotherapeutic agent (temozolomide) acts synergistically to kill glioma stem cells through the HSV-mediated manipulation of DNA damage responses. This strategy is highly efficacious in preclinical models and warrants further clinical study. ${ }^{6}$ The other study is about the immune consequence of cancer cell death induced by a particular OV and a specific chemotherapeutic drug and their potential impact on therapeutic outcome. It is thought that the cure of particularly aggressive malignancies may require induction of immunogenic cancer cell death (ICD), which leads to oncolysis and presentation of tumor-associated antigens (TAAs) coupling with exposure/release of danger signal molecules, such as cell surface exposure of calreticulin, and release of ATP, and high-mobility group box protein B1 from dying tumor cells, eliciting a potent antitumor immunity. Angelova et al. ${ }^{7}$ have shown complementary induction of ICD by oncolytic parvovirus H-1PV and gemcitabine in pancreatic cancer cells. These results warrant further studies of such combination therapy in animal tumor models.

The potential synergy between OVs and radiation may depend on the specific virus, specific genetic mutations of the cancer and type/dose of radiation. A recent study has found that synergistic cytotoxicity of radiation and oncolytic Lister strain vaccinia virus (V) to BRAF mutant melanoma depends on JNK and tumor necrosis factor alpha (TNF- $\alpha$ ) signaling. ${ }^{8}$ The virus GLV-1h68 combined with radiotherapy significantly increased cytotoxicity and apoptosis relative to either single agent in ${ }^{\mathrm{V} 600 \mathrm{D}} \mathrm{BRAF} /{ }^{\mathrm{V} 600 \mathrm{E}} \mathrm{BRAF}$ mutant melanoma cells in vitro and in vivo. The mechanism of enhanced cytotoxicity from the combination was independent of viral replication but due to attenuation of JNK, p38 and ERK MAPK phosphorylation specifically in BRAF mutant cells.

\section{COMBINATION WITH VASCULATURE-TARGETING OR PHOTODYNAMIC THERAPY}

Many studies have shown that the OV alone or OV armed with angiogenesis inhibitors can work to inhibit tumor growth and metastasis. ${ }^{9,10}$ Photodynamic therapy (PDT) is a clinically approved regimen for cancer treatment. PDT induced vascular disruption and resulted in higher viral titers in the tumor compared with the untreated tumor. Combination of PDT with oncolytic VV resulted in growth inhibition of primary and metastatic tumors compared with either monotherapy. ${ }^{11}$ PDT enhances viral replication in the tumor mass and the overall therapeutic efficacy. Intriguingly, Garg et al. ${ }^{12}$ have demonstrated that hypericin-based PDT (Hyp-PDT) is a bona fide ICD inducer. Hyp-PDT triggered a signaling pathway that leads to calreticulin (CRT) exposure and ATP release by cancer cells, a type of ICD, thus potentially eliciting antitumor immune responses. $^{12}$ Therefore, multi-mechanistic pathways, including oncolysis, antiangiogenesis and antitumor immunity, all contribute to therapeutic efficacy for oncolytic-PDT therapy.

\section{COMBINATION WITH TARGETED THERAPY ON SPECIFIC SIGNALING PATHWAYS}

Cancer cells often display alteration/mutations in specific signaling pathways, which have been utilized for targeting and selective replication of OVs. ${ }^{13}$ It is possible that inhibition/promotion of specific signaling pathways may promote OV viral replication and oncolysis, or simply promote cancer cell death, thus leading to improved therapeutic efficacy. As an example, MG18L, an oncolytic HSV with US3 deletion, replicated well in glioblastoma stem cells (GSCs), and had antiglioblastoma activity in mice. PI3K/ Akt inhibitors displayed significant but variable antiproliferative activities in GSCs. The combination of the two synergized in killing GSCs and glioma cells, but not human astrocytes, through enhanced induction of apoptosis. Somehow, synergy was independent of inhibitor sensitivity. In vivo, the combination significantly prolonged survival of mice. This study establishes a novel therapeutic strategy using the ability of an oncolytic HSV to manipulate critical oncogenic pathways to sensitize cancer cells to a molecularly targeted drug. ${ }^{14}$ OVT of gallbladder cancer with 
OVs with cancer vaccines, adoptive immune cell transfer, immunostimulatory cytokines, Toll-like receptor ligands, and have obtained promising results. We would like to discuss a few recent significant studies. We have utilized Th1-Tc1-attracting chemokine-armed OV for combined oncolytic immunotherapy in a model of colorectal cancer. ${ }^{17}$ In the late stage of cancer, OV alone is insufficient to break immune tolerance in the highly immunosuppressive tumor microenvironment. It might be possible to induce an immune response if OV is combined with an ICDinducing chemotherapeutic drug. ${ }^{18}$ The combination of a HSV-1 OV with mitoxantrone provided significant survival benefit to $\mathrm{BALB} / \mathrm{c}$ mice bearing Her2/neu TUBO-derived tumors. This protection was mediated by increased infiltration of neutrophils and tumor antigen-specific $\mathrm{CD}^{+}{ }^{+} \mathrm{T}$ cells into tumor. This study suggests that OVs in combination with an ICD-inducing drug can enhance cancer immunogenicity, breaking immunologic tolerance established toward these tumor antigens. ${ }^{18}$ Song and coworkers ${ }^{19}$ have made seminal discovery using an oncolytic $\mathrm{W}$ that encodes a secretory bispecific T-cell engager consisting of two single-chain variable fragments specific for $\mathrm{CD} 3$ and the tumor cell surface antigen EphA2 (termed EphA2-T-cell engagerarmed VV). The function of this virus is twofold: first acting as an $\mathrm{OV}$ and second as a virally secreted molecule to activate T cells. The OV plus human T cells had potent antitumor activity in a lung cancer xenograft model in comparison to either monotherapy. Thus, this armed oncolytic VV may represent a promising new approach to improve OVT. The fourth study is combination of OV with immune checkpoint blockade. Recent investigations led to two important concepts that, first, preexisting lymphocytic infiltration of tumors is associated with superior prognostic outcomes in a variety of cancers; second, immune checkpoint blockade could work together with strategies that induce tumor inflammation. Built on these notions, Zamarin et al. ${ }^{20}$ have just shown that localized OVT using an oncolytic Newcastle disease virus (NDV) can overcome systemic tumor resistance to immune checkpoint blockade immunotherapy in the immunosuppressive B16 melanoma model. More specifically, localized intratumoral injection of NDV induces inflammatory responses, leading to lymphocytic infiltrates and antitumor effect in distant tumors without distant virus spread. Localized NDV injection and systemic CTLA-4 blockade led to rejection of pre-established distant tumors and protection from tumor rechallenge in poorly immunogenic tumor models, irrespective of tumor cell line sensitivity to NDVmediated lysis. This study provides a strong rationale for clinica trials on such combination therapies in human cancer patients. ${ }^{20}$

myxoma virus can be enhanced by hyaluronan $(\mathrm{HA}) .^{15}$ The recent study has shown that HA interacts with CD44 to activate the Akt signaling pathway, which increases rates of oncolysis. HA also enhanced the MMP-9 secretion, which contributes to collagen IV degradation, thus the viral diffusion and spread.

Cancer cells often overexpress antiapoptotic proteins and thus are resistant to apoptosis-inducing agents including a number of OVs. Smac mimetic compounds (SMCs) are a class of drugs that sensitize cancer cells to apoptosis by counteracting the activity of inhibitor of apoptosis proteins. Inflammatory cytokines can induce cancer cell death when combined with SMC. Korneluk and colleagues $^{16}$ reasoned that SMCs would synergize with agents that stimulate a potent yet safe 'cytokine storm'. They demonstrated that OVs and adjuvants such as poly (I:C) and CpG induce bystander death of cancer cells treated with SMCs. This is mediated by cytokines interferon- $\beta$, TNF- $\alpha$ and/or TNF-related apoptosis-inducing ligand. This combinatorial treatment resulted in tumor regression and extended survival in two murine tumor models. $^{16}$

\section{COMBINATION WITH IMMUNOTHERAPY}

As we have reviewed recently, OVT itself is a novel form of immunotherapy. ${ }^{1,2}$ Many studies have focused on combination of

\section{PERSPECTIVES}

The rationales of combination therapy are multifacious. First, specific OVs induce direct oncolysis of the cancer cells, usually via specific cell death pathways with one as a predominant pathway. Cancer cells have evolved multiple mechanisms to evade cell death, inhibiting one or more specific pathways of cell death. This provides a rationale for combining another therapeutic regimen to kill cancer cells through another death pathway, thus functionally additively or synergistically. Second, cancer cell death has immunological consequences. OVs often induce ICD, releasing/ presenting danger signals, and present TAAs, which are critical initial steps in activating DCs and eliciting adaptive antitumor immunity. Thus, it is logical to combine OV with some other form of immunotherapy to enhance the overall efficacy. Third, metastases are the main reason for cancer fatality. Many standard therapeutic regimens cannot target micrometastases and cancer stem cells effectively, resulting in cancer relapse. Immunotherapy is very effective in inhibiting distant metastases. A variety of anticancer cytotoxic treatments, such as a variety of modes of chemotherapy, radiation, PDT, all lead to multimodal ICD as a consequence (Figure 1). Thus it is only rational to integrate 
immunotherapy into most therapeutic regimens in order to maximize the therapeutic efficacy, and prolong the disease progression-free survival. Some of these strategies have generated exciting and promising results in tumor models, and it is high time to test them in clinical trials.

\section{CONFLICT OF INTEREST}

DLB serves as a member of the Scientific Advisory Board and is shareholder of Jennerex BioTherapeutics, a company focusing on development of OVs. ZSG declares no conflict of interest.

ZS Guo and DL Bartlett Department of Surgery, The University of Pittsburgh Cancer Institute, University of Pittsburgh School of Medicine, Pittsburgh, PA, USA E-mail: guozs@upmc.edu or bartlettdl@upmc.edu

\section{REFERENCES}

1 Bartlett DL, Liu Z, Sathaiah M, Ravindranathan R, Guo Z, He Y et al. Oncolytic viruses as therapeutic cancer vaccines. Mol Cancer 2013; 12: 103.

2 Guo ZS, Liu Z, Bartlett DL. Oncolytic immunotherapy: dying the right way is a key to eliciting potent antitumor immunity. Front Oncol 2014; 4: 74.

3 Chiocca EA, Rabkin SD. Oncolytic viruses and their application to cancer immunotherapy. Cancer Immunol Res 2014; 2: 295-300.

4 Ottolino-Perry K, Diallo JS, Lichty BD, Bell JC, McCart JA. Intelligent design: combination therapy with oncolytic viruses. Mol Ther 2010; 18: 251-263.

5 Chase M, Chung RY, Chiocca EA. An oncolytic viral mutant that delivers the CYP2B1 transgene and augments cyclophosphamide chemotherapy. Nat Biotechnol 1998; 16: 444-448.

6 Kanai R, Rabkin SD, Yip S, Sgubin D, Zaupa CM, Hirose Y et al. Oncolytic virusmediated manipulation of DNA damage responses: synergy with chemotherapy in killing glioblastoma stem cells. J Natl Cancer Inst 2012; 104: 42-55.

7 Angelova AL, Grekova SP, Heller A, Kuhlmann O, Soyka E, Giese T et al. Complementary induction of immunogenic cell death by oncolytic parvovirus $\mathrm{H}-1 \mathrm{PV}$ and gemcitabine in pancreatic cancer. J Virol 2014; 88: 5263-5276.

8 Kyula JN, Khan AA, Mansfield D, Karapanagiotou EM, McLaughlin M, Roulstone V et al. Synergistic cytotoxicity of radiation and oncolytic Lister strain vaccinia in
(V600D/E)BRAF mutant melanoma depends on JNK and TNF-alpha signaling. Oncogene 2014; 33: 1700-1712.

9 Angarita FA, Acuna SA, Ottolino-Perry K, Zerhouni S, McCart JA. Mounting a strategic offense: fighting tumor vasculature with oncolytic viruses. Trends $\mathrm{Mol}$ Med 2013; 19: 378-392.

10 Gil M, Seshadri M, Komorowski MP, Abrams SI, Kozbor D. Targeting CXCL12/ CXCR4 signaling with oncolytic virotherapy disrupts tumor vasculature and inhibits breast cancer metastases. Proc Natl Acad Sci USA 2013; 110: E1291-E1300.

11 Gil M, Bieniasz M, Seshadri M, Fisher D, Ciesielski MJ, Chen Y et al. Photodynamic therapy augments the efficacy of oncolytic vaccinia virus against primary and metastatic tumours in mice. Br J Cancer 2011; 105: 1512-1521.

12 Garg AD, Krysko DV, Verfaillie T, Kaczmarek A, Ferreira GB, Marysael T et al. A novel pathway combining calreticulin exposure and ATP secretion in immunogenic cancer cell death. EMBO J 2012; 31: 1062-1079.

13 Guo ZS, Thorne SH, Bartlett DL. Oncolytic virotherapy: molecular targets in tumorselective replication and carrier cell-mediated delivery of oncolytic viruses. Biochim Biophys Acta 2008; 1785: 217-231.

14 Kanai R, Wakimoto H, Martuza RL, Rabkin SD. A novel oncolytic herpes simplex virus that synergizes with phosphoinositide 3-kinase/Akt pathway inhibitors to target glioblastoma stem cells. Clin Cancer Res 2011; 17: 3686-3696.

15 Weng M, Gong W, Ma M, Chu B, Qin Y, Zhang M et al. Targeting gallbladder cancer: oncolytic virotherapy with myxoma virus is enhanced by rapamycin in vitro and further improved by hyaluronan in vivo. Mol Cancer 2014; 13: 82

16 Beug ST, Tang VA, LaCasse EC, Cheung HH, Beauregard CE, Brun J et al. Smac mimetics and innate immune stimuli synergize to promote tumor death. Nat Biotechnol 2014; 32: 182-190.

17 Li J, O'Malley M, Urban J, Sampath P, Guo ZS, Kalinski P et al. Chemokine expression from oncolytic vaccinia virus enhances vaccine therapies of cancer. Mol Ther 2011; 19: 650-657.

18 Workenhe ST, Pol JG, Lichty BD, Cummings DT, Mossman KL. Combining oncolytic HSV-1 with immunogenic cell death-inducing drug mitoxantrone breaks cancer immune tolerance and improves therapeutic efficacy. Cancer Immunol Res 2013; 1: 309-319.

19 Yu F, Wang X, Guo ZS, Bartlett DL, Gottschalk SM, Song XT. T-cell engager-armed oncolytic vaccinia virus significantly enhances antitumor therapy. Mol Ther 2014; 22: $102-111$.

20 Zamarin D, Holmgaard RB, Subudhi SK, Park JS, Mansour M, Palese P et al. Localized oncolytic virotherapy overcomes systemic tumor resistance to immune checkpoint blockade immunotherapy. Sci Transl Med 2014; 6: 226ra32. 